\title{
ON FINITE GROUPS CONTAINING THREE $C$-SUBGROUPS
}

\author{
ZVI ARAD AND PAMELA FERGUSON
}

\begin{abstract}
A finite group $G$ has a self-centralization system of type $\left(2\left|A_{1}\right|\right.$, $\left.4\left|A_{2}\right|, 4\left|A_{3}\right|\right)$ if $G$ contains three nonconjugate $C C$-subgroups $A_{1}, A_{2}, A_{3}$, such that $\left|N_{G}\left(A_{1}\right)\right|=2\left|A_{1}\right|,\left|N_{G}\left(A_{2}\right)\right|=4\left|A_{2}\right|,\left|N_{G}\left(A_{3}\right)\right|=4\left|A_{3}\right|$. The authors prove that if a finite group $G$ has a self-centralization system of type $\left(2\left|A_{1}\right|, 4\left|A_{2}\right|, 4\left|A_{3}\right|\right)$ and $|G|<3\left|A_{1}\right|^{2}\left|A_{2}\right|^{2}\left|A_{3}\right|^{2}$, then $G$ has a nilpotent normal subgroup $N$ such that $G / N$ is isomorphic to $\mathrm{Sz}(q)$ for suitable $q$.
\end{abstract}

Introduction. A proper subgroup $A$ of a group $G$ is called a $C C$-subgroup if $C_{G}(a)<A$ for all $a \in A^{\sharp}$. In this paper we shall consider a group $G$ with a set $A_{1}$, $A_{2}, A_{3}$ of nonconjugate $C C$-subgroups. If $\left[N_{G}\left(A_{i}\right): A_{i}\right]=d_{i}>1,1<i<3$, we shall describe this as a self-centralization system of type $\left(d_{1}\left|A_{1}\right|, d_{2}\left|A_{2}\right|, d_{3}\left|A_{3}\right|\right)$ or $\left(d_{1}, d_{2}, d_{3}\right)$ for short.

The fact is that many simple groups have self-centralization systems. For example, $J_{4}, J_{11}$ and $M_{22}$ have types (22(23), 28(29), 10(31), 12(37), 14(43)), (6(7), 10(11), 6(19)) and (4(5), 3(7), 5(11)), respectively. Groups of Ree type have type $(6,6)$.

Several authors have studied groups with self-centralization systems of type (2). (See [6], [7] and [8].) It is an open question of Higman [7] whether or not there are finite simple groups of type $(2,4,4)$ other than the Suzuki groups. Usami [10] answers this question under restrictions on the order of the $C C$-subgroups of $G$ and the assumption that if $r$ is an odd prime then an $S_{r}$-subgroup of $G$ is abelian and $|G| \leqslant 3\left|A_{1}\right|^{2}\left|A_{2}\right|^{2}\left|A_{3}\right|^{2}$.

In this paper we shall prove the following theorem:

THEOREM A. If $G$ is a finite simple group with a self-centralization system of type $\left(2\left|A_{1}\right|, 4\left|A_{2}\right|, 4\left|A_{3}\right|\right)$ and $|G| \leqslant 3\left|A_{1}\right|^{2}\left|A_{2}\right|^{2}\left|A_{3}\right|^{2}$, then $G$ is isomorphic to one of the Suzuki groups.

Theorem A has the following corollary.

COROLlaRY A. If $G$ is a finite group with a self-centralization system of type $\left(2\left|A_{1}\right|, 4\left|A_{2}\right|, 4\left|A_{3}\right|\right)$ and $|G| \leqslant 3\left|A_{1}\right|^{2}\left|A_{2}\right|^{2}\left|A_{3}\right|^{2}$, then $G$ has a nilpotent normal subgroup $N$ such that $G / N$ is isomorphic to $S z(q)$ for suitable $q$.

Many papers appear in the literature which classify groups which have a character $\Lambda$ of small degree relative to the orders of some subgroups of $G$. In a forthcoming paper, the authors will use Theorem A to classify all groups of type

Received by the editors May 21, 1979.

AMS (MOS) subject classifications (1970). Primary $20 \mathrm{C} 15$. 
$\left(2 n_{1}, 4 n_{2}, 4 n_{3}\right)$ which have a character $\Lambda$ of relatively small degree compared to $n_{1} n_{2} n_{3}$.

1. Our notations are taken from [5] and character notation from [6]. If $K$ is a nonempty set, let $|K|$ denote the number of elements in $K$. If $p$ is a prime, then $G_{p}$ denotes a Sylow $p$ subgroup of $G$.

Let $G \in$ Hypothesis $\mathrm{A}$ if $\mathrm{G}$ is a finite simple group with a self-centralization system of type $\left(2\left|A_{1}\right|, 4\left|A_{2}\right|, 4\left|A_{3}\right|\right)$.

Let $\left|A_{1}\right|=n_{1},\left|A_{2}\right|=n_{2}$ and $\left|A_{3}\right|=n_{3}$ where $n_{2}<n_{3}$. Let $t_{1}=\left(n_{1}-1\right) / 2$, $t_{2}=\left(n_{2}-1\right) / 4$ and $t_{3}=\left(n_{3}-1\right) / 4$.

Let $G \in$ Hypothesis A, then Theorem 5.1 [7] implies $G$ has one class of involutions. If $\tau$ is a fixed involution, then by conjugacy we may choose $A_{1}, A_{2}, A_{3}$ to be normalized by $\tau$.

LemMA 1. Let $G \in$ Hypothesis A, then one of the following holds:

(i) $G$ is isomorphic to $S z(q)$.

(ii)(a) $\left(n_{1} n_{2} n_{3}, 3\right)=1$.

(b) $G$ contains elements of order 2, 3, 4, and 6.

Proof. Assume $G$ is not isomorphic to a Suzuki group; then [4] and [3] imply $G$ contains an element of order 6 . Hence $A_{i}$ a $C C$-subgroup for $i=1,2,3$ implies $\left(n_{1} n_{2} n_{3}, 3\right)=1$. Walter [11] implies $G$ contains an element of order 4 .

Let $G \in$ Hypothesis $\mathrm{B}$ if $G \in$ Hypothesis $\mathrm{A}, G$ is not isomorphic to a Suzuki group, and $|G| \leqslant 3\left|A_{1}\right|^{2}\left|A_{2}\right|^{2}\left|A_{3}\right|^{2}$.

Assume $G \in$ Hypothesis $\mathrm{A}$, then $n_{1}>3$. Hence, there are $t_{1}$ irreducible characters $X_{i}, i=1, \ldots, t_{1}$, of $G$ which are associated with $A_{1}$ and one nonprincipal

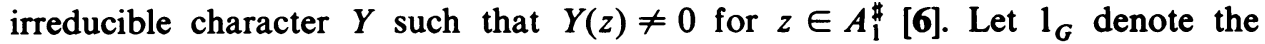
principal character of $G$. Lemma $3[6]$ implies $X_{1}(1)=k n_{1}+2 \varepsilon$ and $Y(1)=k n_{1}+$ $\varepsilon$ where $k$ is a positive integer and $\varepsilon= \pm 1$. Lemma $6[6]$ implies $|G|=$ $n_{1} X_{1}(1) Y(1)\left(\ln _{1}+1\right)$ where $l$ is a nonnegative integer.

We incorporate some properties of $X_{1}$ and $Y$ into Lemma 2.

Lemma 2. Assume $G \in$ Hypothesis A and let $T=\cup_{g \in G}\left(A_{1}^{\#}\right)^{8}$, then the following hold:

(i) If $x \in G-T$, then $X_{1}(x)$ and $Y(x)$ are integers, and $X_{1}(x) Y(x)$ is a nonnegative integer.

(ii) $\Sigma_{x \in G-T} X_{1}(x) Y(x)=|G| / n_{1}$.

(iii) $\left|C_{G}(\tau)\right|^{2}=\left(\ln _{1}+1\right)(Y(1)-Y(\tau))^{2}$.

(iv) $|Y(\tau)| \leqslant k+\varepsilon$.

(v) $(Y(1)-Y(\tau))^{2} \geqslant k^{2}\left(n_{1}-1\right)^{2}$.

(vi) $\left(n_{2} n_{3}, X_{1}(1)\right) \neq 1$. If $l=0$, then $n_{2} n_{3} \mid X_{1}(1)$.

Proof. Since $Y$ is the only nonprincipal nonexceptional irreducible character of $G$ which does not vanish on $A_{1}^{\#}, Y$ is equal to all its algebraic conjugates. Hence $Y(z)$ is an integer for all $z \in G$. Lemma 3 [6] implies if $x \in G-T$, then $X_{1}(x)=Y(x)+\varepsilon$. Hence $X_{1}(x)$ is an integer. Now $X_{1}(x) Y(x)=Y^{2}(x)+\varepsilon Y(x)$ and $Y(x)$ an integer implies (i). 
The orthogonality nelations imply $\Sigma_{G} X_{1}(g) Y(g)=0$. Now $\Sigma_{T} X_{1}(g) Y(g)=$ $\left(|G| / 2 n_{1}\right)\left(\Sigma_{g \in A^{\ddagger}} \zeta_{1}(g)\right)$ where $\zeta_{1}$ is an irreducible character of $N_{G}\left(A_{1}\right)$ associated with $X_{1} \cdot \Sigma_{A \ddagger} \zeta_{1}(g)=-2$; hence $\Sigma_{G-T} X_{1}(x) Y(x)=|G| / n_{1}$ and (ii) is proved.

Lemma 6 [6] implies (iii).

$N_{G}\left(A_{1}\right)$ a Frobenius group of order $2 n_{1}$ implies there are $t_{1}$ irreducible characters $\zeta_{i}$ of $N_{G}(A)$ such that $\zeta_{i}(1)=2$ and $\zeta_{i}$ vanishes off $A_{1}$. Let $\lambda$ be a faithful linear character of $N_{G}\left(A_{1}\right) / A_{1}$, then $\left\{\lambda, \lambda^{2}, \zeta_{i} \mid i=1, \ldots, t_{1}\right\}$ are all the irreducible characters of $N_{G}\left(A_{1}\right)$. Since $Y$ is a nonexceptional character, it follows that $\left.Y\right|_{N_{G}\left(A_{1}\right)}=$ $r \lambda^{2}+s \lambda+\sum_{i=1}^{t_{1}} k \zeta_{i}$ where $r+s=k+\varepsilon$. Now $\tau \in N\left(A_{1}\right)$ implies $Y(\tau)=r-s$; hence $|Y(\tau)| \leqslant k+\varepsilon$. Parts (iv) and (v) of the lemma follow.

Now $\Sigma_{A_{1}^{*}} X_{1}(g) \overline{X_{1}(g)}=\varepsilon^{2} \Sigma_{A_{1}^{*}} \zeta_{1}(g) \overline{\zeta_{1}(g)}=2 n_{1}-4$. Thus $\left(X_{1}, X_{1}\right)=|G|$ implies $\Sigma_{G-T} X_{1}(x) \overline{X_{1}(x)}+|G|\left(2 n_{1}-4\right) / 2 n_{1}=|G|$. Hence $\Sigma_{G-T} X_{1}(x) \overline{X_{1}(x)}=$ $2|G| / n_{1}$. Suppose $\left(X_{1}(1), n_{2} n_{3}\right)=1 . X_{1}$ is a nonexceptional character for $i=2$ or 3 . Hence, if $t_{i} \geqslant 2$ for $i=2$ or 3 , it follows that $\left|X_{1}(x)\right|=1$ for all $x \in A_{i}^{\#}$. If $t_{2}=1$, then $n_{2}=5$ and all elements of order 5 are again conjugate. Hence (i) implies $\left|X_{i}(x)\right|=1$ for all $x \in A_{2}^{\#}$. Thus $\left(n_{2} n_{3}, X_{1}(1)\right)=1$ implies

$$
|G|\left(n_{2}-1\right) / 4 n_{2}+|G|\left(n_{3}-1\right) / 4 n_{3}<|G| 2 / n_{1} \text {. }
$$

Now $n_{1} \geqslant 5$ implies $1 / 10<1 / 2-2 / n_{1}<1 / 4 n_{2}+1 / 4 n_{3}<2 / 4 n_{2}$. Hence $n_{2}<$ 5 , which is a contradiction. Therefore, $\left(X_{1}(1), n_{2} n_{3}\right) \neq 1$.

If $l=0$, then $\left|G_{2}\right| \mid X_{1}(1)$ or $\left|G_{2}\right| \mid Y(1)$. Theorem $17.4[4]$ implies $X_{1}(\tau)=0$ or $Y(\tau)=0$. Lemma 3 [6] implies $Y(1)-Y(\tau)=X_{1}(1)-X_{1}(\tau)$, hence (iii) implies $\left|C_{G}(\tau)\right|=X_{1}(1)$ or $Y(1)$. Now $\left(n_{2} n_{3}, X_{1}(1)\right) \neq 1$ implies $\left|C_{G}(\tau)\right|=Y(1)$ so that $n_{2} n_{3} \mid X_{1}(1)$.

\section{LeMmA 3. Assume $G \in$ Hypothesis $\mathrm{B}$, then $n_{1}>11$ and $n_{2} n_{3} \mid X_{1}(1)$.}

Proof. Suppose $n_{1}>7$, then Lemma 1(ii)(a) implies $n_{1}>11$. Suppose $t_{i}>2$ for $i=2$ or 3 , then $\left\{X_{j} \mid j=1, \ldots, t_{1}\right\}$ does not contain any exceptional characters associated with $N\left(A_{i}\right)$. It follows that $X_{j}(z)=X_{1}(z)$ for $z \in A_{i}^{\#}$ and $t_{1} X_{1}(z)^{2}<3$. Now $n_{1} \geqslant 11$ and Lemma 2(i) imply $X_{1}(z)=0$. Hence $n_{i} \mid X_{1}(1)$. Suppose $n_{2}=5$, and let $z \in A_{2}^{\#}$. Since $X_{j}(z)=X_{1}(z)$ for $j=1, \ldots, t_{1}$, the orthogonality relations imply $5 X_{1}^{2}(z) \leqslant t_{1} X_{1}^{2}(z) \leqslant\left|C_{G}(z)\right|-1=4$. Lemma $2(\mathrm{i})$ implies $X_{1}(z)=0$. Thus $n_{1}>7$ implies $n_{2} n_{3} \mid X_{1}(1)$.

We will assume $n_{1} \leqslant 7$ and obtain a contradiction. Let $x_{i}, i=1, \ldots, t_{1}$, be representatives in $A_{1}^{\#}$ of the conjugacy classes of $A_{1}^{\#}$. Similarly let $y_{j}, j=1, \ldots, t_{2}$, and $z_{\tilde{k}}, \tilde{k}=1, \ldots, t_{3}$, be representatives of the conjugacy classes of $A_{2}^{\#}$ and $A_{3}^{\#}$. Let $B=\left\{x_{i}, y_{j}, z_{\tilde{k}} \mid i, j, \tilde{k}\right\}$, then $|B|=b=t_{1}+t_{2}+t_{3}$.

If $C_{G}(\tau) u C_{G}(\tau) \cap C_{G}(\tau) v C_{G}(\tau) \neq \varnothing$ for distinct elements $u, v \in B$, then there are elements $w$ and $h \in C_{G}(\tau)$ such that $v=h u w$. Conjugation by $\tau$ yields $v^{-1}=v^{\tau}=(h u w)^{\tau}=h u^{-1} w$. It follows that $v=\left(v^{\tau}\right)^{-1}=\left(h u^{-1} w\right)^{-1}=w^{-1} u h^{-1}$. Hence, $v^{2}=\left(w^{-1} u h^{-1}\right)(h u w)=w^{-1} u^{2} w$. Since all elements in $B$ have odd order, $v^{2}=u^{2 w}$ implies that $|\langle u\rangle|=|\langle v\rangle|=k$. Now $k$ odd implies $v=\left(v^{2}\right)(k+1) / 2=$ $\left(u^{2}\right)^{w((k+1) / 2)}=\left(u^{2(k+1) / 2}\right)^{w}=u^{w}$. This contradicts the fact that $u$ and $v$ are not conjugate. Therefore, $C_{G}(\tau) u C_{G}(\tau) \cap C_{G}(\tau) v C_{G}(\tau)=\varnothing$ if $u$ and $v$ are distinct. If $w \in C_{G}(\tau) \cap C_{G}(\tau)^{u}$ where $u \in B$, then $w \in C_{G}(\tau) \cap C_{G}\left(\tau \tau^{u}\right)=C_{G}(\tau) \cap C_{G}\left(u^{2}\right)$ 
$=1$. Therefore $\left|C_{G}(\tau) u C_{G}(\tau)\right|=\left|C_{G}(\tau)\right|^{2}$ if $u \in B$. It follows that $b\left|C_{G}(\tau)\right|^{2}<|G|$. Lemma 6 [6] implies that $|G|(Y(1)-Y(\tau))^{2}=n_{1}\left(k n_{1}+\varepsilon\right)\left(k n_{1}+2 \varepsilon\right)\left|C_{G}(\tau)\right|^{2}$. Hence, $b\left|C_{G}(\tau)\right|^{2} \leqslant|G|$ implies that $b(Y(1)-Y(\tau))^{2}<n_{1}\left(k n_{1}+\varepsilon\right)\left(k n_{1}+2 \varepsilon\right)$. Lemma 2(v) now implies

$$
b k^{2}\left(n_{1}-1\right)^{2} \leqslant n_{1}\left(k n_{1}+2 \varepsilon\right)\left(k n_{1}+\varepsilon\right) .
$$

Lemma 2(iii) implies that $n_{2} n_{3} \mid X_{1}(1) Y(1)$.

Suppose $n_{1}=5$, Lemma 1 implies $n_{2} \geqslant 13$ and $n_{3}>17$, hence $b>9$. Inequality (3.1) implies $19 k^{2}-75 k \varepsilon-10<0$. Hence $\varepsilon=1$ and $k<4$. Direct computation of $X_{1}(1) Y(1)$ for $\varepsilon=1$ and $1 \leqslant k \leqslant 4$ imply there are no $n_{2}$ and $n_{3}$ such that $n_{2} n_{3} \mid X_{1}(1) Y(1)$. Hence $n_{1}>5$.

Suppose $n_{1}=7$. If $k \leqslant 3$, then direct computation for $\varepsilon= \pm 1$ implies there are no $n_{2}$ and $n_{3}$ such that $n_{2} n_{3} \mid X_{1}(1) Y(1)$. Hence we may assume $k>4$. Inequality (3.1) implies $b \leqslant 11$, hence $\left(n_{2}-1\right) / 4+\left(n_{3}-1\right) / 4 \leqslant 11-3=8$. Calculation implies $\left(n_{2}, n_{3}\right)=(5,13),(5,17)$ or $(13,17)$.

If $\left(n_{2}, n_{3}\right)=(13,17)$, then $b=10$ and (3.1) implies $17 k^{2}-147 k \varepsilon-14<0$. Hence $\varepsilon=1$ and $k<9$. Direct computation implies $13 \cdot 17 \nmid X_{1}(1) Y(1)$ for $\varepsilon=1$ and $k \leqslant 9$. Hence $\left(n_{2}, n_{3}\right)=(5,13)$ or $(5,17)$.

Suppose $l \neq 0$. Lemma 2(iii) implies $l 7+1$ is a square, hence $l>5$. Let $T \in\left\{X_{1}, Y\right\}$ where $n_{3} \mid T(1)$; then $T(1)=f n_{3}$. Since $G$ satisfies Hypothesis B, $|G|<3 \cdot 7^{2} \cdot 5^{2} \cdot n_{3}^{2}$. Now $3 \cdot 5^{2} \cdot 7^{2} \cdot n_{3}^{2} \geqslant|G| \geqslant 7\left(f n_{3}-1\right)^{2}(5 \cdot 7+1)$ implies $f$ <4. If $n_{3}=17$ and $T=Y$, then $17 f= \pm 1(\bmod 7)$ implies $f=2$ and $Y(1)=34$ $=5 \cdot 7-1$. Hence $X_{1}(1)=33$ and $5 \nmid Y(1) X_{1}(1)$. If $n_{3}=17$ and $T=X_{1}$, then $17 f= \pm 2(\bmod 7)$ implies $f=3$ and $X_{1}(1)=7 \cdot 7+2$. Hence $Y(1)=50$, but $n_{2}=5$ implies $50 \nmid|G|$. Therefore $n_{3} \neq 17$. Similar calculations imply $n_{3} \neq 13$.

Hence, we may assume $l=0$. Lemma 2 (vi) implies $X_{1}(1)=e n_{2} n_{3}$. Now $7\left(e n_{2} n_{3}-1\right)\left(e n_{2} n_{3}\right) \leqslant|G| \leqslant 3 \cdot 7^{2} \cdot 5^{2} \cdot n_{3}^{2}$ implies $e<4$. Since 4||$G \mid$ and $\left|G_{2}\right| \mid Y(1), e=1$ or 3 . However $17 \cdot 5=1(\bmod 7)$ and $3 \cdot 17 \cdot 5=3(\bmod 7)$ imply $n_{3} \neq 17$. If $n_{3}=13$, then $5 \cdot 13 \cdot 3=6(\bmod 7)$ implies $X_{1}(1)=5 \cdot 13$ and $Y(1)=64$. Hence $(|G|, 3)=1$ and $G \notin$ Hypothesis $B$.

Lemma 4. Assume $G \in$ Hypothesis $B$. Let $U=\{Z \mid Z$ an irreducible character of $G$ and $\left.n_{1} n_{2} n_{3} \nmid Z(1)\right\}$, then $|U|<4+t_{1}+t_{2}+t_{3}$.

Proof. If $n_{2}>5$, Lemma 4 [10] and Lemmas 1 and 3 imply the result. Hence we may assume $n_{2}=5$. Let $x_{i}, i=2,3$, denote elements of $A_{2}^{\#}$ and $A_{3}^{\#}$ respectively. Lemma 3 implies $n_{2} n_{3} \mid X_{1}(1)$. Lemma 3 [6] implies $Y\left(x_{i}\right)=-\varepsilon$ for $i=2$, 3. Since all elements of order 5 are conjugate, the orthogonality relations imply there are 3 irreducible characters $Z_{1}, Z_{2}$ and $Z_{3}$ of $G$ such that

$$
\left|Z_{i}\left(x_{2}\right)\right|=1 \text {. }
$$

If $V=\left\{1_{G}, Y, Z_{1}, Z_{2}, Z_{3}\right\}$, then $|V|=5$ and $V$ contains all the irreducible characters of $G$ whose degrees are not divisible by 5 .

Let $\left\{X_{3, j} \mid j=1, \ldots, t_{3}\right\}$ be the exceptional characters of $G$ associated with $N_{G}\left(A_{3}\right)$. The orthogonality relations imply there are 2 irreducible characters $Z_{5}, Z_{4}$ 
of $G$ such that $\left|Z_{5}\left(x_{3}\right)\right|=\left|Z_{4}\left(x_{3}\right)\right|=1$. If $W=\left\{1_{G}, Y, Z_{4}, Z_{5}, X_{3 j} \mid j=1, \ldots, t_{3}\right\}$, then $|W|=t_{3}+4$ and $W$ contains all the irreducible characters of $G$ whose degrees are not divisible by $n_{3}$.

Now $Y\left(x_{2}\right) Y\left(x_{3}\right)+1_{G}\left(x_{2}\right) 1_{G}\left(x_{3}\right)=2$ and the orthogonality relations imply

$$
\sum_{i=1}^{3} Z_{i}\left(x_{2}\right) Z_{i}\left(x_{3}\right)=-2
$$

Hence $Z_{i}\left(x_{3}\right) \neq 0$ for some $i=1,2$, or 3. Now $X_{3, j}\left(x_{2}\right)=X_{3,1}\left(x_{2}\right)$ for $j=$ $1, \ldots, t_{3}$; hence $\left\{Z_{i} \mid i=1,2,3\right\} \cap\left\{X_{3, j} \mid j=1, \ldots, t_{3}\right\} \neq \varnothing$ implies $\left(n_{3}-1\right) / 4$ $=3$ and $\left\{Z_{i} \mid i=1,2,3\right\}=\left\{X_{3 j} \mid j=1,2,3\right\}$. Hence $U=\left\{1_{G}, Y, X_{i}, X_{3, j}, Z_{4}, Z_{5} \mid i\right.$ $\left.=1, \ldots, t_{1}, j=1, \ldots, t_{3}\right\}$ and $|U|=3+t_{1}+t_{2}+t_{3}$. If $\left\{Z_{i} \mid i=1,2,3\right\} \cap$ $\left\{X_{3, j} \mid j=1, \ldots, t_{3}\right\}=\varnothing$, then (4.1) and (4.2) imply $\left\{Z_{4}, Z_{5}\right\} \subset\left\{Z_{1}, Z_{2}, Z_{3}\right\}$. Hence $U=\left\{1_{G}, Y, Z_{1}, Z_{2}, Z_{3}, X_{i}, X_{3, j} \mid i=1, \ldots, t_{1}, j=1, \ldots, t_{3}\right\}$ so that $|U|=$ $t_{1}+t_{2}+t_{3}+4$.

Lemma 5. Assume $G \in$ Hypothesis $\mathrm{B}$, then $|G|>\left(n_{1} n_{2} n_{3}\right)^{2}$. If $r$ is an odd prime which is relatively prime to $3 n_{1} n_{2} n_{3}$ and $r|| G \mid$, then $r=5$ and $G_{5}$ is an elementary abelian CC-subgroup of order 25.

Proof. Let $t$ be the number of conjugacy classes of elements in $G$ which do not meet $\left(A_{1} \cup A_{2} \cup A_{3}\right)^{\#}$. Lemma 1 (ii) implies $t=5+j$ where $j>0$. Lemma 4 implies there are at least $j+1$ irreducible characters $Z$ of $G$ such that $n_{1} n_{2} n_{3} \mid Z(1)$. Hence $(j+1)\left(n_{1} n_{2} n_{3}\right)^{2}<|G|$ implies $|G|>\left(n_{1} n_{2} n_{3}\right)^{2}$ and $j<1$.

Let $r$ be as in the hypothesis, then $j=1$. Hence, centralizers of elements of order $r$ are $G_{r}$ groups. Therefore, $G_{r}$ is a $C C$-subgroup of $G$ [9]. Since all elements of $G_{r}^{\#}$ are conjugate, $G_{r}$ is elementary abelian, and $C_{G}\left(G_{r}\right)=G_{r}$. Now $j=1$ and Lemma 1 imply $G$ contains no elements of order 12 or 8 .

$N_{G}\left(G_{r}\right)$ is a Frobenius group with $G_{r}$ as a Frobenius kernel. Let $L$ be the Frobenius complement. Clearly $|L|=\left|G_{r}^{\sharp}\right|$. Since $L$ is a Frobenius complement, an $S_{2}$-subgroup of $L$ is cyclic or generalized quaternion and the Sylow subgroups of $L$ of odd prime order are cyclic. In this case, the highest power of 2 dividing $|L|$ is 2 or 4 or 8 by Lemma 1 . If an odd prime $r^{\prime}$ divides $|L|$, it is well known that 2||$Z(L) \mid$. Hence $r^{\prime}=3$. Hence $|L|=2,4,8,6,12$, or 24 . Consequently, $\left|G_{r}\right|=3,5$, $9,7,13$, or $5^{2}$. The hypothesis implies $\left(\left|G_{r}\right|, 3\right)=1$; hence $\left|G_{r}\right|=5,7,13,5^{2}$.

On the other hand, let $y$ be an element of order $r$. Then $N_{G}(\langle y\rangle) / C_{G}(y)$ is a cyclic group of order $r-1$ since the elements of order $r$ form a single class. Therefore, the possibilities of the order of a cyclic subgroup of even order in $G$ are $2,4,6$ and $\left|G_{r}\right|=5,7$ or $5^{2}$. Let $x$ be an element of order $r$. The class equation implies

$$
|G| \geqslant 1+\frac{|G|}{\left|G_{r}\right|}+\frac{|G|\left(n_{1}-1\right)}{2 n_{1}}+\frac{|G|\left(n_{2}-1\right)}{4 n_{2}}+\frac{|G|\left(n_{3}-1\right)}{4 n_{3}}
$$

Hence $1 / 2 n_{1}+1 / 4 n_{2}+1 / 4 n_{3} \geqslant 1 /\left|G_{r}\right|$. Lemma 3 implies $n_{1}>11$. Now $n_{2}>5$ and $n_{3} \geqslant 13$ imply $\left|G_{r}\right|>7$. 
Lemma 6. Assume $G \in$ Hypothesis $\mathrm{B}$, then the following hold:

(i) $X_{1}(1)=n_{2} n_{3}$.

(ii) $\ln 1+1=n_{1}+1$ or $3 n_{1}+1$.

(iii) $\ln _{1}+1=2^{2 a} 3^{2 b}$ where $a$ is a positive integer and $b$ is a nonnegative integer.

Proof. Lemma 3 implies $X_{1}(1)=e n_{2} n_{3}$, where $e$ is a positive integer.

Suppose $l=0$. As in the proof of Lemma 2, we see that $\left|C_{G}(\tau)\right|=Y(1)$. Lemma 5 implies $\left(n_{1} n_{2} n_{3}\right)^{2}<n_{1}\left(e n_{2} n_{3}\right)\left(e n_{2} n_{3}+1\right)$, hence $e>1$. Lemma 1 implies 3||$C_{G}(\tau) \mid$, hence $\left(X_{1}(1), Y(1)\right)=1$ implies $\left|G_{3}\right| \mid Y(1)$. Therefore $X_{1}(1)=e n_{2} n_{3}$ where $e$ is odd, $\left(e, 3 n_{1} n_{2} n_{3}\right)=1$, and $e>1$. Lemma 5 implies $\left(\left|C_{G}(\tau)\right|, 5\right)=5$ and $X_{1}(1)=25 n_{2} n_{3}$. Hence $X_{1}(1)=1(\bmod 4)$. Since $4 \mid Y(1), Y(1)=X_{1}(1)-\varepsilon$ implies $\varepsilon=1$. This contradicts Theorem A [2]. Hence $l \geqslant 1$.

Since $l \neq 0$,

$$
n_{1}\left(e n_{2} n_{3}\right)\left(e n_{2} n_{3}-1\right)\left(\ln _{1}+1\right) \leqslant|G| \leqslant 3\left(n_{1} n_{2} n_{3}\right)^{2}
$$

implies $l e^{2}<4$. Hence $e=1$ and $l \leqslant 3$. Lemma 2 (iii) implies $\ln _{1}+1$ is a square, hence $l=1$ or 3 . Lemmas 1,5 and 2(iii) imply $\ln _{1}+1=2^{2 a} 3^{2 b}$, where $a \geqslant 1$.

Lemma 7. Assume $G \in$ Hypothesis $\mathrm{B}$, then $\ln _{1}+1=2^{2 a} 3^{2 b}$ where $a$ and $b$ are positive integers.

Proof. Lemma 6(iii) implies $a$ is a positive integer. Suppose $b=0$. Lemmas 1 and 5 imply $\left|C_{G}(\tau)\right|=\left|G_{2}\right| 3^{c}$ where $c \geqslant 1$. Lemma 6 implies $X_{1}(1)=n_{2} n_{3}$. Hence $\ln _{1}+1=2^{2 a}$ implies $\left|G_{3}\right| \mid Y(1)$. Lemma 2(iii) implies $\left(Y(1)_{2}\right)^{2}\left(\ln _{1}+1\right)^{2} 3^{2 c}=$ $\left|C_{G}(\tau)\right|^{2}=\left(\ln _{1}+1\right)(Y(1)-Y(\tau))^{2}$. Valuation Theory implies $|Y(\tau)|_{2}=Y(1)_{2}$ and $|Y(\tau)|_{3}=3^{c}$. Lemma 2(iv) now implies

$$
\left|C_{G}(\tau)\right|=|Y(\tau)|_{2}|Y(\tau)|_{3}\left(\ln _{1}+1\right) \leqslant|Y(\tau)|\left(\ln _{1}+1\right)<(k+1)\left(\ln _{1}+1\right) .
$$

Lemma 2(iii) and (v) imply $\left|C_{G}(\tau)\right|^{2} \geqslant k^{2}\left(n_{1}-1\right)^{2}\left(\ln _{1}+1\right)$. It follows that

$$
(k+1)^{2}(\ln +1)^{2} \geqslant\left|C_{G}(\tau)\right|^{2} \geqslant k^{2}\left(n_{1}-1\right)^{2}(\ln +1) .
$$

Now $l \leqslant 3$ and $k \geqslant 1$ imply $4\left(3 n_{1}+1\right) \geqslant\left(n_{1}-1\right)^{2}$. Hence $n_{1}<15$. Lemmas 1 and 3 imply $n_{1}=11$ or 13 . However $n_{1}+1 \neq 2^{2 a}$ if $l=1$ or 3 and $n_{1}=11$ or 13 .

Proof of Theorem A. We will assume $G \in$ Hypothesis B and obtain a contradiction. Lemma 7 implies $\left|G_{2}\right| \nmid Y(1)$, hence Theorem 17.4 [1] implies there is an element $y$ such that $|\langle y\rangle|=2^{k}>1$ and $Y(y) \neq 0$. Since $Y$ is integer valued, $Y\left(y^{i}\right)=Y(y)$ if $(i, 2)=1$. Lemma 6 implies $X_{1}(1)=n_{2} n_{3}$. Lemma 2 implies $X_{1}(z)$ is an integer for $z \in G-\cup_{g}\left(A_{1}\right)^{g}$. Hence $\left(n_{2} n_{3}, 2\right)=1$ implies $X_{1}\left(y^{i}\right) \neq 0$ for any $i$.

Let $R=\left\{y^{g} \mid g \in G\right\}, n=|G| /\left|G_{2}\right|$ and $U_{R}=\left\{x \mid x^{n} \in R\right\}$. Clearly $\cup_{R}$ is a union of conjugacy classes. Since $(n, 8)=1$, there is an odd integer $i_{0}$ such that $y^{i_{0} n}=y$. Let $z_{1}=y^{i_{0}}$, Lemma 2(i) implies $X_{1}\left(z_{1}\right) Y\left(z_{1}\right)$ is a positive integer. Let $z_{1}, z_{2}, \ldots, z_{t}$ be representatives of the conjugacy class in $\cup_{R}$. Now $X_{1} Y$ is a character of $G$, hence Theorem 17.3 [1] implies

(a) $(|G|, n)^{-1} \Sigma_{\cup_{R}} X_{1}(x) Y(x)=c$ where $c$ is an algebraic integer. Since $\cup_{R} \leqslant G$ - $T$, Lemma 2(i) and $X_{1}\left(z_{1}\right) Y\left(z_{1}\right) \neq 0$ imply $c$ is a positive rational number. Hence $c$ is a positive integer. 


$$
\left|G_{2}\right|=Y(1)_{2}\left(\ln _{1}+1\right)_{2} \text { and (a) imply (b) } \Sigma_{\cup_{R}} X_{1}(x) Y(x)=c(|G|, n)=
$$
$c|G| /\left|G_{2}\right|=c|G| / Y(1)_{2}\left(\ln _{1}+1\right)_{2}$. Now

$$
\sum_{\bigcup_{R}} X_{1}(x) Y(x)=\sum_{i=1}^{t} \frac{X_{1}\left(z_{i}\right) Y\left(z_{i}\right)|G|}{\left|C_{G}\left(z_{i}\right)\right|}
$$

and the orthogonality relations imply

$$
Y(1) \mid \frac{X_{1}\left(z_{i}\right) Y\left(z_{i}\right)|G|}{\left|C_{G}\left(z_{i}\right)\right|} \quad \text { for } i=1, \ldots, t \text {. }
$$

Equation (b) now implies

$$
Y(1) \mid \frac{c|G|}{Y(1)_{2}\left(\ln _{1}+1\right)_{2}}=\frac{c|G|}{|G|_{2}} .
$$

Hence $c=Y(1)_{2} d$ where $d$ is a positive integer. Thus

$$
\sum_{\cup_{R}} X_{1}(x) Y(x)=d G /\left(\ln _{1}+1\right)_{2} \text {. }
$$

Lemma 2(ii) now implies $d G /\left(\ln _{1}+1\right)_{2}<|G| / n_{1}$ so that $\left(\ln _{1}+1\right)_{2}>n_{1}$. Lemma 7 implies $l n_{1}+1 \geqslant 9\left(\ln _{1}+1\right)_{2}>9 n_{1}$. Hence $l>3$ which contradicts Lemma 6 .

\section{REFERENCES}

1. W. Feit, Characters of finite groups, Benjamin, New York, 1967.

2. P. Ferguson, On finite simple groups with self-centralization systems of type (2(n)), Proc. Amer. Math. Soc. 72 (1978), 443-444.

3. L. R. Fletcher, B. Stellmacher and W. B. Stewart, Endliche Gruppen, die kein Element der Ordnung 6 enthalten, Quart J. Math. Oxford Ser. 28 (1977), 143-154.

4. G. Glauberman, Factorizations in local subgroups of finite groups, CBMS Regional Conf. Ser. Math., no. 33, Amer. Math. Soc., Providence, R. I., 1977.

5. D. Gorenstein, Finite groups, Harper and Row, New York, 1968.

6. K. Harada, A characterization of groups $\operatorname{LF}(2, q)$, Illinois J. Math. 11 (1967), 647-659.

7. G. Higman, Odd characterizations of finite simple groups, Lecture Notes, University of Michigan, Ann Arbor, Michigan, 1968.

8. W. B. Stewart, Groups having strongly-self-centralizing 3-centralizers, Proc. London Math. Soc. (3) 26 (1973), 653-680.

9. M. Suzuki, Finite groups with nilpotent centralizers, Trans. Amer. Math. Soc. 99 (1961), 425-470.

10. Y. Usami, A characterization of the Suzuki groups, Natur. Sci. Rep. Ochanomizu Univ. 26 (1975), 13-29.

11. J. Walther, The characterization of finite groups with Abelian 2-subgroups, Ann. of Math. 89 (1969), 405-514.

12. J. S. Williams, A sufficient condition on centralizers for a finite group to contain a proper CCT subgroup, J. Algebra 42 (1976), 549-556.

Department of Mathematics, University of Miami, Coral Gables, Florida 33124 